\title{
A Case of Polymyositis Associated with Metastasis Anaplastic Thyroid Cancer
}

\author{
Mnif F* , Boujelben K, Ben Salah D, Loukil F, Elleuch M, Hadjkacem F and Abid M
}

Endocrinology-Diabetology Department, CHU Hédi Chaker, Sfax, Tunisia

"Corresponding author: Mnif F, Endocrinology-Diabetology Department, CHU Hédi Chaker, Sfax, Tunisia, Tel: +216-74

244 221; E-mail: fatmamnif05@yahoo.fr

Received: August 22, 2019; Accepted: August 29, 2019; Published: September 05, 2019

\begin{abstract}
Polymyositis (PM), an autoimmune inflammatory myopathy, can be manifested as a paraneoplastic syndrome (PS). Reports on the association of PM with thyroid cancer are very few. This report presents the case of a 69-year-old woman who had a history of autoimmune hypothyroidism, treated with thyroxin. She developed progressive proximal muscle weakness. Clinical features, laboratory results and muscle biopsy led us to the diagnosis of polymyositis. Few days later, after corticoid therapy, she showed the symptoms of a thyroid carcinoma, most likely anaplastic. Although the association between malignancy and polymyositis remains controversial, to our knowledge this is the first report of anaplastic thyroid carcinoma (ATC) occurring in association with polymyositis and auto-immune hypothyroidism. As a result, thyroid studies should be performed in cancer investigations in PM cases.
\end{abstract}

Keywords: Polymyositis; Anaplastic thyroid cancer; Hypothyroidism

\section{Background}

Polymyositis (PM) and dermatomyositis (DM) are idiopathic inflammatory myopathies with cutaneous characteristics and muscle manifestations [1,2]. Both disorders are predominantly affecting adults [1,3]. The diagnosis is based on a combination of clinical, laboratory and muscle biopsy findings [4]. Idiopathic inflammatory myopathy (IIM) is reportedly associated with various types of malignancy, with DM patients facing a greater risk [5,6]. We present a case of a woman who developed PM before the diagnosis of an anaplastic thyroid carcinoma.

\section{Case Report}

A 69-year-old woman was admitted to our hospital for proximal muscle weakness, myalgias of the shoulder and neck muscles and progressive difficulty walking uphill and standing up from a chair. A prior diagnoses of Hashimoto thyroiditis (HT) and multinodular goitrous was made 10 years ago for which the patient was treated with thyroxin. Her family medical history was negative for neuromuscular diseases.

Citation: Mnif F, Boujelben K, Ben Salah D, et al. A Case of Polymyositis Associated with Metastasis Anaplastic Thyroid Cancer. Clin Case Rep Open Access. 2019;2(3):133.

(C)2019 Yumed Text. 
www.yumedtext.com | September-2019

Clinically she had no fever, arthralgia or arthritis, cough, dyspnea, rash, paresthesias, abdominal pain, or other symptoms. Her physical examination was revealed for 2/5 muscle strength in the deltoid muscle, $3 / 5$ in the biceps and triceps muscles and $4 / 5$ in the lower extremities. There were no skin lesions. She has euthyroid with thyroxin supplementation and has a multinodular goiter.

At the present admission, laboratory investigation showed elevated serum level of creatine phospokinase (CPK) 3776 IU/L, as well as an elevation of alpha 2 globulinemia at $10.2 \mathrm{~g} / \mathrm{l}$, and increased aminotransaminase serum level (7-fold elevation of ASAT and 3-fold elevation of ALAT).

No cholestasis was observed. A thyroid Stimulating Hormone (TSH) value was at normal range (0.95 UI/l) with thyroxin supplementation. We performed an open biopsy of her muscle, which on microscopically light demonstrated an inflammatory myositis, compatible with PM, as well as mild fiber atrophy and necrosis. No evidence of vascularitis or other metabolic myopathy was detected.

C 5 and B 9, a pathological hallmark of complex membrane attack, was shown in the muscle membrane in the perifascular area as well as in degenerating muscle fibers (FIG. 1).
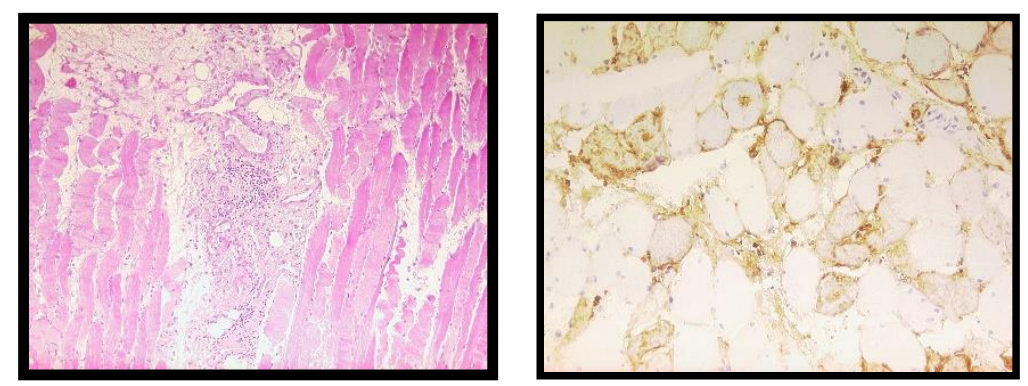

FIG. 1. Representative images showing histological features of skeletal muscles biopsies: Muscle biopsy data: C5B9 immunocytochemistry membrane attack complex is very positive.

Antinuclear antibodies were not detected with standard immunofluorescence as well as the myositis-specific antibody antiJo-1, rheumatoid factor, anti-DNA were negative.

The anti- mitochondria and smooth muscle antibodies were also negative. The possibility of a paraneoplasic myopathy was discussed. We performed abdominal and pelvic tomography which turned up to be normal. Neck ultrasound demonstrated a multinodular goiter and presence of several nodules; the greater were respectively $18 \mathrm{~mm}$ and $21 \mathrm{~mm}$. The patient was started on corticoids bolus for 3 days and $1 \mathrm{mg} / \mathrm{kg} / \mathrm{d}$ after and intravenous polyvalent immunoglobulin (TEGELINE).

Under this treatment, there was improvement in the proximal weakness and incomplete normalization of the muscle enzymes. One month after the original presentation, she came back with acute increase in the volume of the goiter without muscles weakness. Clinically there was a firm, immobile right mass over the neck without dysphagia, stidor or hoarseness. 
www.yumedtext.com | September-2019

Computed tomography of the neck demonstrated a large infiltrating thyroid mass measured $60 \mathrm{~mm}$ occupying most of the right lobe of the thyroid with tracheal displacement. Fine-needle aspiration revealed anaplastic thyroid carcinoma. Immunohistochemistry using cytokeratin CK5, CK6, and CK7 were positive but were negative after using thyrocalcitonin (TCT) and thyroglobulin (TG).

The fluorine-18; 2-floro-D-glucose positron emission tomography (18F-FDG PET) scan was performed; it showed multiple intensive uptakes in the thyroid mass, sternum and liver (FIG. 2).

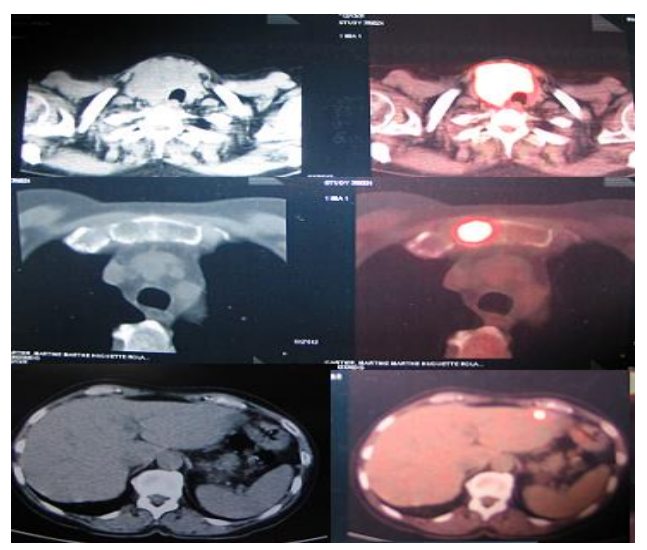

FIG. 2. The fluorine-18; 2-floro-D-glucose positron emission tomography (18F-FDG PET) scan showed multiple intensive uptakes in the thyroid mass, sternum and liver.

Under the diagnosis of anaplastic thyroid carcinoma with metastasis, we decided to treat the patient with systemic chemotherapy (ADRIBLASTINE-CISPLATYL) with external cervico-mediastinal radiotherapy (65 gray). After chemoradiotherapy, clinical symptoms and all laboratory abnormalities markedly improved. During more than 10 years of followup, the patient remained in good condition, without PM symptoms, or cancer relapse.

\section{Discussion}

Anaplastic thyroid carcinoma (ATC) is one of the most aggressive malignancies with poor prognosis [7]. Although ATC accounts for only $1 \%$ to $2 \%$ of the thyroid cancers, it is responsible for more than half of the death attributed to thyroid cancer [8]. ATC is rarely associated with paraneoplastic events. The relationship between malignant disease and myositis was first described in 1916 [9].

Since then, several authors confirmed the association between malignancy and IIM with a frequency ranging $6 \%-40 \%$, but its meaning and significance have remained unclear. Presumably, it is the result of an immune reaction against antigens common for muscle and neoplastic cells or some paraneoplastic syndrome underlying mechanism.

Recent report confirmed that the risk of cancer in IIM is concentrated among patients with DM. The association between DM and cancer was enhanced by its temporal relationship ( $<1$ year) in $87.5 \%$ in these cases patients with malignancy-associated DM were more frequently male and over age 45 and less likely to have interstitial lung disease [10]. 
www.yumedtext.com | September-2019

In several studies, the most common cancer types noted were adenocarcinomas, which accounted for $70 \%$ of all associated tumors in both DM and PM patients [10,11]. The spectrum of malignancies associated with DM or PM parallels the distribution in the general population with the possible exceptions of an increased frequency of cervical, lung, ovarian, pancreatic, bladder, and gastric carcinomas and with non-Hodgkin lymphoma [11].

We report a case of PM associated with thyroid metastasis anaplastic carcinoma. This association is rare and in our knowledge this is the first report of this association. The other case in the literature was often DM rather than PM [5,12-14]. The spectrum of thyroid cancer was dominated by the papillary carcinoma [5,12-14], one case of plasmocytoma [15] and one case of anaplatic non metastatic carcinoma [16].

Our patient was diagnosed with PM based on clinical presentation, elevated CK and muscle biopsy results. Thyroid cancer succeeded the development of PM in our case.

In conclusion, this is the second report of PM associated with anaplastic thyroid cancer. Awareness of a potential PM complication is important because it can be confused with other causes of muscle weakness in the case of malignancy such as myasthenic syndromes, hypothyroidism. Keeping in mind that PM may be associated with thyroid cancer, extensive diagnostic work-up to exclude neoplastic lesions should be performed.

\section{REFERENCES}

1. Dalakas MC, Hohlfeld R. Polymyositis and dermatomyositis. Lancet. 2003;362(9388):971-82.

2. Findlay AR, Goyal NA, Mozaffar T. An overview of polymyositis and dermatomyositis. Muscle Nerve. 2015;51(5):638-56

3. Kalliabakos D, Pappas A, Lagoudianakis E, et al. A case of polymyositis associated with papillary thyroid cancer: a case report. Cases J. 2008;1(1):289.

4. Sasaki H, Kohsaka H. Current diagnosis and treatment of polymyositis and dermatomyositis. Mod Rheumatol. 2018;28(6):913-21.

5. Fujita M, Hatachi S, Yagita M. Dermatomyositis associated with thyroid cancer. Rheumatol Int. 2008;29(2):203-5.

6. Qiang JK, Kim WB, Baibergenova A, et al. Risk of Malignancy in Dermatomyositis and Polymyositis. J Cutan Med Surg. 2017;21(2):131-6.

7. Molinaro E, Romei C, Biagini A, et al. Anaplastic thyroid carcinoma: from clinicopathology to genetics and advancedtherapies. Nat Rev Endocrinol. 2017;13(11):644-60.

8. Janz TA, Neskey DM, Nguyen SA, et al. Is the incidence of anaplastic thyroid cancer increasing: A population based epidemiology study. World J Otorhinolaryngol Head Neck Surg. 2018;5(1):34-40.

9. Stertz G. Polymyositis. Berl Klin Wochenschr. 1916;53:489.

10. Antiochos BB, Brown LA, Li Z, et al. Malignancy is associated with dermatomyositis but not polymyositis in Northern New England, USA. J Rheumatol. 2009;36(12):2704-10.

11. Hill CL, Zhang Y, Sigurgeirsson B, et al. Frequency of specific cancer types in dermatomyositis andpolymyositis: a population-based study. Lancet. 2001;357(9250):96-100. 
www.yumedtext.com | September-2019

12. Lee JH, Kim SI. A case of dermatomyositis associated with papillary cancer of the thyroid gland. Clin Rheumatol. 2005;24(4):437-8.

13. Nagashima T, Onishi S, Kamata Y, et al. Dermatomyositis associated with thyroid cancer: a paraneoplastic syndrome? Rheumatol Int. 2009;29(10):1261-2.

14. Shah M, Shah NB, Moder KG, et al. Three cases of dermatomyositis associated with papillary thyroid cancer. Endocr Pract. 2013;19(6):e154-7.

15. Caron P, Lassoued S, Thibaut I, et al. Thyroid plasmacytoma with dermatomyositis and palmar fasciitis. J Rheumatol. 1989;16(7):997-9.

16. Smith C, Staniland J. A difficult case of inflammatory myositis. Age Ageing. 2003;32(3):351-2. 